\title{
Spontaneous and Amplified Radiation at the Initial Stage of a SASE FEL
}

\author{
Zhirong Huang*, Kwang-Je Kim ${ }^{\text {a }}$

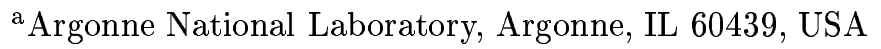

At the initial stage of a self-amplified spontaneous emission (SASE) free-electron laser (FEL), spontaneous undulator radiation in certain experimental configurations can dominate the amplified signal over an extended undulator distance. In this paper we study both the spontaneous and the amplified radiation in the framework of the paraxial wave equation and determine the transition from the dominance of spontaneous emission to exponential amplification. We compare theoretical expectations with SASE simulation codes GINGER and GENESIS.

\section{Introduction}

In many self-amplified spontaneous emission (SASE) free-electron laser (FEL) experiments radiation near the fundamental wavelength is collected through a large opening angle and without a monochromator [1-3]. This results in a large background of spontaneous undulator radiation in the detected signal, together with the amplified signal. Correctly describing the detected radiation can help characterize the FEL performance and the electron beam properties. In this paper, we investigate the validity of the paraxial wave equation used in FEL theory and simulations in describing spontaneous undulator radiation at the initial stage of a SASE FEL. Comparing the spontaneous radiation power with the amplified one under these experimental conditions, we find the transition from the dominance of spontaneous emission to exponential amplification can occur at an undulator distance much longer than the first two power gain lengths that produce the effective start-up noise. These results are compared with SASE simulation codes GINGER and GENESIS.

\section{Analysis at the initial stage of a SASE FEL}

The Maxwell equation for the transverse electric field $E_{x}$ is

$$
\begin{array}{r}
{\left[\left(\frac{1}{c} \frac{\partial}{\partial t}\right)^{2}-\left(\frac{\partial}{\partial z}\right)^{2}-\frac{\partial^{2}}{\partial \boldsymbol{x}^{2}}\right] E_{x}(\boldsymbol{x}, z, t)} \\
=-\frac{1}{\epsilon_{0} c^{2}}\left[\frac{\partial J_{x}}{\partial t}+c^{2} \frac{\partial \rho_{e}}{\partial x}\right],
\end{array}
$$

where $\boldsymbol{x}=(x, y)$ represents the transverse coordinates, $\epsilon_{0}$ is the permittivity of free space, $J_{x}$ is the transverse current density, and $\rho_{e}$ is the charge density. The Fourier representation of the electric field in both $t$ and $\boldsymbol{x}$ is

$$
\begin{gathered}
A_{\nu}(\boldsymbol{\phi}, z)=\frac{1}{\lambda^{2}} \int d^{2} x e^{-i k \boldsymbol{x} \cdot \boldsymbol{\phi}} \int \frac{d t}{\sqrt{2 \pi}} e^{i \omega(t-z / c)} \\
\times E(\boldsymbol{x}, z, t)
\end{gathered}
$$

where $\omega=\nu \omega_{1}=2 \pi c / \lambda=k c$, the fundamental undulator frequency is $\omega_{1}=4 \pi c \gamma^{2} /\left[\lambda_{u}(1+\right.$ $\left.\left.\left.K^{2} / 2\right)\right]\right), \lambda_{u}=2 \pi / k_{u}$ is the undulator period, and $K$ is the undulator parameter. Assuming that $A_{\nu}$ varies slowly with $z$, Eq. (1) becomes the paraxial wave equation for $A_{\nu}$

$$
\begin{aligned}
& {\left[\frac{\partial}{\partial z}+i \frac{k \phi^{2}}{2}\right] A_{\nu}(\phi, z)=\frac{1}{2 \epsilon_{0} c^{2}} \int d^{2} \phi} \\
& \times e^{i k \boldsymbol{x} \cdot \boldsymbol{\phi}} \int \frac{d \omega}{\sqrt{2 \pi}} e^{i \omega(t-z / c)}\left[-J_{x}+c \phi_{x} \rho_{e}\right] .
\end{aligned}
$$

Solving this equation for a single electron source would lead to the angular-spectral distribution of 
the $\sigma$-polarization for undulator radiation (e.g., Eq.(4.23) of Ref. [4]). In general, we cannot drop the charge density term of Eq. (3) because $J_{x} \sim \frac{K c}{\gamma} \cos \left(k_{u} z\right) \rho_{e}$ and $\phi_{x} \sim 1 / \gamma$ for spontaneous undulator radiation. However, since the FEL interaction is driven resonantly by the current density term and occurs in a radiation angle much smaller than $1 / \gamma$ for $\omega \sim \omega_{1}$ or $\nu \sim 1$, the charge density term of Eq. (3) is normally dropped in FEL theory and simulations. In the absence of the FEL interaction this approximation leads to [5]

$$
\begin{aligned}
A_{\nu}(\boldsymbol{\phi}, z) \approx & -\exp \left(-\frac{i k \phi^{2} z}{2}\right) \sum_{j} e^{-i \nu \theta_{j}} \\
& \times U_{\nu}\left(\boldsymbol{\phi}, \eta_{j}, \boldsymbol{x}_{j}, \boldsymbol{p}_{j}\right) .
\end{aligned}
$$

Here $\theta_{j}, \eta_{j}, \boldsymbol{x}_{j}, \boldsymbol{p}_{j}$ represent the initial phase, the relative energy, the transverse position and the angular divergence of the $j^{\text {th }}$ electron.

$$
\begin{aligned}
& U_{\nu}(\boldsymbol{\phi}, \eta, \boldsymbol{x}, \boldsymbol{p})=u_{1} e^{-i k \boldsymbol{\phi} \cdot \boldsymbol{x}} \int_{0}^{z} d s e^{i(\Delta \nu-2 \eta \nu) k_{u} s} \times \\
& \exp \left[\frac{i k}{2} \int_{0}^{s} d s^{\prime}\left(\left(\boldsymbol{\phi}-\boldsymbol{p}\left(s^{\prime}\right)\right)^{2}+k_{\beta}^{2} \boldsymbol{x}^{2}\left(s^{\prime}\right)\right)\right],
\end{aligned}
$$

where $u_{1}=\frac{e K[\mathrm{JJ}]}{4 \sqrt{2 \pi} \epsilon_{0} \gamma \lambda^{2} c}$, [JJ] is the Bessel function factor, and $k_{\beta}$ is the betatron wavenumber. For parallel electrons $(\boldsymbol{p}=0)$ without any focusing $\left(k_{\beta}=0\right)$, we have

$$
\left|U_{\nu}\right|=u_{1} z \frac{\sin \Phi}{\Phi},
$$

where

$$
\Phi=\frac{z}{2}\left[(\Delta \nu-2 \eta \nu) k_{u}+\frac{k}{2} \phi^{2}\right] .
$$

From Eq. (4), the angular distribution of the spectral power near the fundamental frequency is approximately given by

$$
\begin{aligned}
& \frac{d^{3} P_{1}}{d \omega d^{2} \phi} \approx \frac{2 \epsilon_{0} c \lambda^{2}}{T}\left\langle A_{\nu}^{*}(\phi, z) A_{\nu}(\phi, z)\right\rangle \\
& =\frac{e^{2}}{4 \pi \epsilon_{0} c} \frac{N_{e}}{T}\left(\frac{\omega}{\omega_{1}}\right)^{2}\left(\frac{\gamma K[\mathrm{JJ}]}{1+K^{2} / 2}\right)^{2} N_{u}^{2}\left(\frac{\sin \Phi}{\Phi}\right)^{2},
\end{aligned}
$$

where $T$ is the pulse duration, and $N_{e}$ is the total number of electrons. We integrate Eq. (7) over all solid angle $\left(\int d^{2} \phi=\pi \int d\left(\phi^{2}\right)\right)$ by using

$$
\frac{\sin ^{2} \Phi}{\Phi^{2}} \approx \frac{1}{N_{u}} \delta\left(\frac{\omega}{\omega_{1}}\left(1+\frac{\gamma^{2} \phi^{2}}{1+K^{2} / 2}-2 \eta\right)-1\right)
$$

for $N_{u} \gg 1$ to obtain the spectral power

$$
\frac{d P_{1}}{d \nu}=N_{u} m c^{2} \frac{I}{I_{A}} \frac{4 \pi^{2} c}{\lambda_{u}}\left(\frac{\gamma K[\mathrm{JJ}]}{1+K^{2} / 2}\right)^{2} \nu,
$$

where $I=e N_{e} / T$ is the peak current and $I_{A}=$ $e c / r_{e}$ is the Alfven current.

Including the charge density term in Eq. (3), one can obtain an exact expression for the $\sigma$ polarization of the fundamental undulator radiation $[6,4]$

$$
\begin{aligned}
\frac{d P_{1 \sigma}}{d \nu}= & N_{u} m c^{2} \frac{I}{I_{A}} \frac{4 \pi^{2} c}{\lambda_{u}}\left(\frac{\gamma K}{1+K^{2} / 2}\right)^{2} \\
& \times \nu\left[\left(\frac{1}{2}-\nu+\frac{3}{2} \nu^{2}\right)+\mathrm{O}\left(K^{2}\right)\right] .
\end{aligned}
$$

Figure 1 shows the frequency dependence of the fundamental undulator radiation given by Eqs. (8) and (9) for $K \ll 1$. We see the paraxial wave equation used in FEL theory and simulations overestimates the spontaneous power everywhere except at the fundamental frequency due to the ignorance of the charge density term. Nevertheless, as frequency approaches the fundamental one or the emission angle is much less than $1 / \gamma$, Eq. (8) is a good approximation of Eq. (9). For a detector that accepts the full angular distribution and a small bandwidth $\Delta \nu_{c}$ just below the fundamental frequency, the spontaneous radiation power expected from both Eqs. (8) and (9) is

$$
P_{1}^{\mathrm{UR}}=N_{u} m c^{2} \frac{I}{I_{A}} \frac{4 \pi^{2} c}{\lambda_{u}}\left(\frac{\gamma K[\mathrm{JJ}]}{1+K^{2} / 2}\right)^{2} \Delta \nu_{c}
$$

to the leading order in $\Delta \nu_{c}$.

Among many transverse modes excited by the spontaneous radiation, a single fundamental mode with the largest exponential growth rate will eventually dominate the FEL signal. Taking into account the energy spread and the emittance 


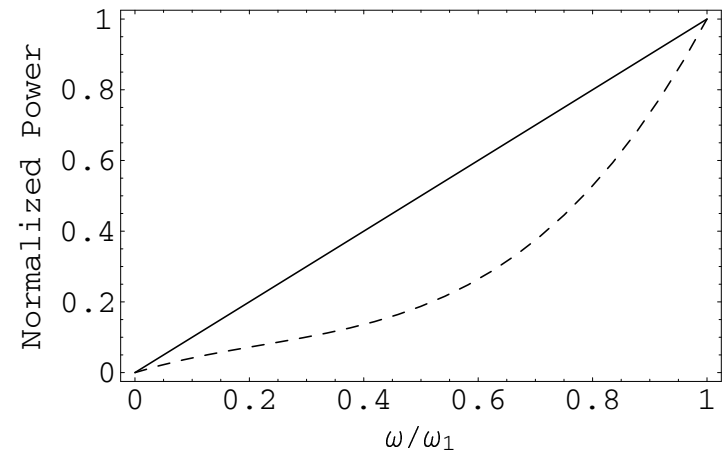

Figure 1. Fundamental undulator radiation spectrum ( $\sigma$-polarization, $K \ll 1)$ as calculated from Eq. (8) (solid line) and Eq. (9) (dashed line).

effects, the power spectrum of the fundamental mode before saturation is

$$
\frac{d P_{\text {fund }}}{d \omega}=g_{A} g_{S} \frac{\rho \gamma m c^{2}}{2 \pi} \exp \left[\frac{z}{L_{G}}-\frac{(\Delta \omega)^{2}}{2 \omega_{1}^{2} \sigma_{\nu}^{2}}\right],
$$

where $g_{A}$ is the input coupling coefficient, $g_{S}$ is the start-up noise coefficient [7], $\rho$ is the FEL scaling parameter [9], $L_{G}$ is the power gain length, and $\omega_{1} \sigma_{\nu}(z)$ is the FEL bandwidth and is a function of $z$. Integration over the SASE spectrum yields

$$
P_{\text {fund }}=g_{A} g_{S} \frac{\rho \gamma m c^{2}}{\sqrt{2 \pi}} \omega_{1} \sigma_{\nu} e^{z / L_{G}}
$$

In Ref. $[8,7]$, the effective start-up power for the fundamental mode is identified as the coherent fraction of the spontaneous radiation in the first two power gain lengths, which is a small fraction of the total spontaneous radiation emitted. Assuming that higher-order transverse modes have negligible growth rates, the exponentially growing fundamental mode will dominate over the spontaneous radiation emitted over all solid angles when $z>z_{t}$, where $z_{t}$ is determined by

$$
\pi \frac{z_{t}}{\lambda_{u}} \frac{I}{I_{A}} \frac{K^{2}[\mathrm{JJ}]^{2}}{1+K^{2} / 2} \Delta \nu_{c}=g_{A} g_{S} \frac{\rho \gamma}{\sqrt{2 \pi}} \sigma_{\nu}\left(z_{t}\right) e^{z_{t} / L_{G}} \text {. }
$$

\section{Comparison with simulation studies}

Two SASE FEL codes GINGER [10] and GENESIS [11] are used for this study. Since GINGER solves for the radiation field that is azymuthally symmetric in transverse coordinates, it excludes most higher-order modes of the spontaneous emission. As a result, GINGER models the central cone of the undulator radiation that possesses the azymuthal symmetry $[6,4]$ and is found to agree with the expected central-cone radiation power at the start-up regime [13]. The threedimensional code GENESIS is used to examine the SASE power emitted in all solid angles. For a limited simulation bandwidth, the GENESIS spectrum at start-up cannot reproduce Eq. (8) due to the effect of aliasing [12]. Nevertheless, we expect the initial GENESIS power within a small bandwidth is comparable to that given by Eq. (10). The output power of GINGER and GENESIS should be comparable when the fundamental mode is dominant (i.e., when $z>z_{t}$ ). We compare $z_{t}$ determined from these codes with that predicted from Eq. (13).

Figure 2 shows such a comparison using the low energy undulator test line (LEUTL) FEL at the Advanced Photon Source operated at $530 \mathrm{~nm}$ [1]. The electron beam energy is $217 \mathrm{MeV}$ with a $0.1 \%$ energy spread. The normalized emittance is $9.3 \mu \mathrm{m}$ in $x$ and $7.6 \mu \mathrm{m}$ in $y$. The bunch profile is assumed to be Gaussian with the rms bunch length $300 \mathrm{fs}$ and the peak current $266 \mathrm{~A}$. The spontaneous undulator radiation, calculated from Eq. (10) by taking $\Delta \nu_{c} \approx 0.12$ to be one half of the simulated bandwidth (the other half is above the fundamental wavelength), is found to be somewhat higher than the initial power of the GENESIS simulation. The results of GINGER and GENESIS converge after $7 \mathrm{~m}$ (including the drift spaces between undulators) as the fundamental mode dominates. Eq. (13) yields $z_{t}=5$ $\mathrm{m}$ using the above beam parameters.

Figure 3 shows another comparison using the Linac Coherent Light Source proposed at the Stanford Linear Accelerator Center for an x-ray FEL at $1.5 \AA$ [14]. The electron beam energy is $14.3 \mathrm{GeV}$ with a $6 \times 10^{-5}$ energy spread. The normalized emittance is $1.5 \mu \mathrm{m}$ in both trans- 


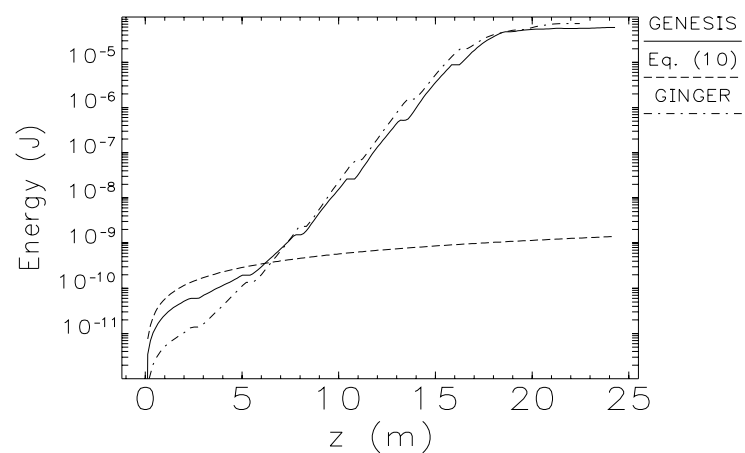

Figure 2. Radiated Energy as a function of the distance for the LEUTL FEL.

verse dimensions. The electron bunch is assumed to be a flat-top profile with $3.4 \mathrm{kA}$ peak current. Since the emittance of the electrons is 4 to 5 times larger than the radiation emittance $\lambda_{1} /(4 \pi)$, many higher-order transverse modes are excited at the initial stage. As a result, the spontaneous undulator radiation contained in all these modes dominates over the fundamental mode over the first half of the entire undulator distance. Equation (13) gives $z_{t} \approx 50 \mathrm{~m}$, while the crossover between GINGER and GENESIS is around $55 \mathrm{~m}$. Higher-order transverse modes in GENESIS are excited by increasing transverse grid points covered by the electron beam [15]. With sufficient transverse grid points, we see that the inital power of GENESIS simulation approaches that given by Eq. (10) for a half of the simulation bandwidth $\Delta \nu_{c}=0.03$.

\section{Acknowledgments}

We thank W. Fawley and S. Reiche for useful discussions on FEL simulations. This work was supported by the U. S. Department of Energy under Contract No. W-31-109-ENG-38.

\section{REFERENCES}

1. S. Milton et al., Science 292, 2037 (2001).

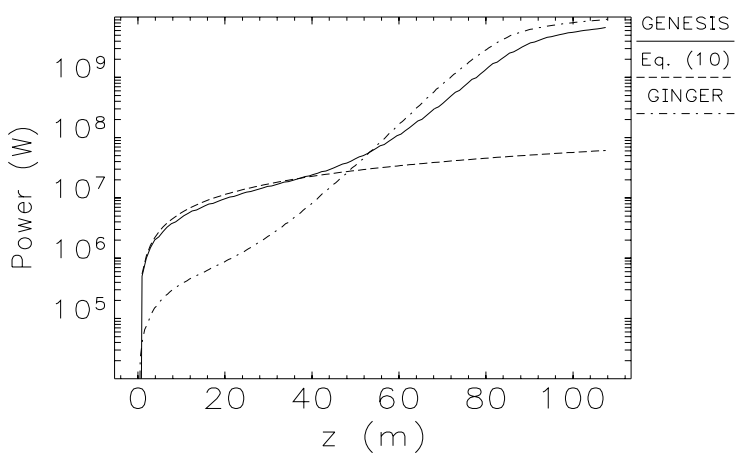

Figure 3. Radiated Power as a function of the distance for the LCLS.

2. V. Ayvazyan et al., Phys. Rev. Lett. 88, 104802-1 (2002).

3. A. Tremaine et al., Phys. Rev. Lett. 88, 204801-1 (2002).

4. K.-J. Kim, in AIP Conference Proceedings 184, M. Month and M. Dienes, eds., 585 (1989).

5. K.-J. Kim, Nucl. Instrum. Methods Phys. Res. A 318, 489 (1992).

6. A. Hofmann, SSRL ACD-Note 38, (1986).

7. Z. Huang and K.-J. Kim, Nucl. Instrum. Methods Phys. Res. A 475, 59 (2001).

8. L.-H. Yu and S. Krinsky, Nucl. Instrum. Methods Phys. Res. A 285, 119 (1989).

9. R. Bonifacio, C. Pellegrini, and L.M. Narducci, Opt. Comm. 50, 373 (1984).

10. W. Fawley, Report LBNl-49625, 2001.

11. S. Reiche, Nucl. Instrum. Methods Phys. Res. A 429, 243 (1999).

12. W.H. Press et al., Numerical Recipes in Fortran 77 (Cambridge Press, Cambridge, 1992).

13. W. Fawley, Phys. Rev. ST Accel. Beams 5, 070701 (2002).

14. Linac Coherent Light Source Design Study Report, SLAC-R-521, 1998.

15. S. Reiche, private communication. 